\title{
MONTE CARLO SIMULATION ON THE EFFECT OF AIR MASS ABOVE THE PRINCESS SIRINDHORN NEUTRONS MONITOR TO ATMOSPHERIC NEUTRONS FROM COSMIC RAYS
}

\author{
Dumrongsak Rodphothong ${ }^{a, *}$ \\ ${ }^{a}$ Department of physics, Ramkhamhaeng University, \\ Ramkhamhaeng Road, Bangkok, Thailand \\ E-mail: dumrongsak.r@rumail.ru.ac.th
}

\begin{abstract}
Neutron monitors are recognized as a key tool for studying the time variations of galactic cosmic rays, especially with regard to solar effects. Cosmic-ray detectors inside the atmosphere do not record cosmic ray particles directly. The so-called primary cosmic rays interact with nuclei in the atmosphere to produce secondary daughter products. Neutron monitors record predominantly the secondary neutrons from these atmospheric showers. The Princess Sirindhorn Neutron Monitor (PSNM) is the first neutron monitor station in Chiang Mai, Northern Thailand, which installed at a high vertical cutoff rigidity of $16.8 \mathrm{GV}$. It provides unique data on the energy dependence of solar synodic variations, cosmic ray anisotropy, Forbush decreases, and solar modulation. In this work we performed Monte Carlo simulations of the atmospheric structure effects on secondary neutron counts above The Princess Sirindhorn Neutron Monitor by using the FLUKA program. These atmospheric processes are well understood, and the response of neutron monitors to these secondary particles is simulated. This work also provides the techniques for further analysis and a better understanding of those effects.
\end{abstract}

40th International Conference on High Energy physics - ICHEP2020

July 28 - August 6, 2020

Prague, Czech Republic (virtual meeting)

\footnotetext{
*Speaker
} 


\section{Introduction}

Cosmic rays are energetic particles from outer space. The most commonly detected primary cosmic rays are protons $(87 \%)$, alpha particles $(12 \%)$ and a small contribution of heavier nuclei (1\%). [4] They move along the interplanetary magnetic field to earth. When a cosmic ray strikes an air molecule in the earth's atmosphere, it can produce a cascade of secondary particles, which we can detect by using a neutron monitor. The Princess Sirindhon Neutron Monitor (PSNM), the first neutron monitor station in Thailand, is shown in Figure 1. It was set up at the Doi Inthanon mountain summit in Chiang Mai province to measure secondary neutrons count rate related to the flux of cosmic rays in space. Doi Inthanon is the highest point in Thailand, 2565 meters above sea level; therefore, the count rate is about six times higher than that at sea level. When cosmic rays enter the earth's magnetic field, some of them with lower energy will move along the earth's magnetic field to near a magnetic pole. Cosmic rays with higher energy will pass through the magnetic field. We use the cutoff rigidity, a momentum per charge, to separate the cosmic rays by their rigidity thresholds for penetration of the cosmic rays through earth's geomagnetic field to the atmosphere, in units of GV (GV stands for Giga Volt). The cutoff rigidity of Thailand is $16.8 \mathrm{GV}$, which the highest cutoff rigidity of any neutron monitor station in the world. [2]

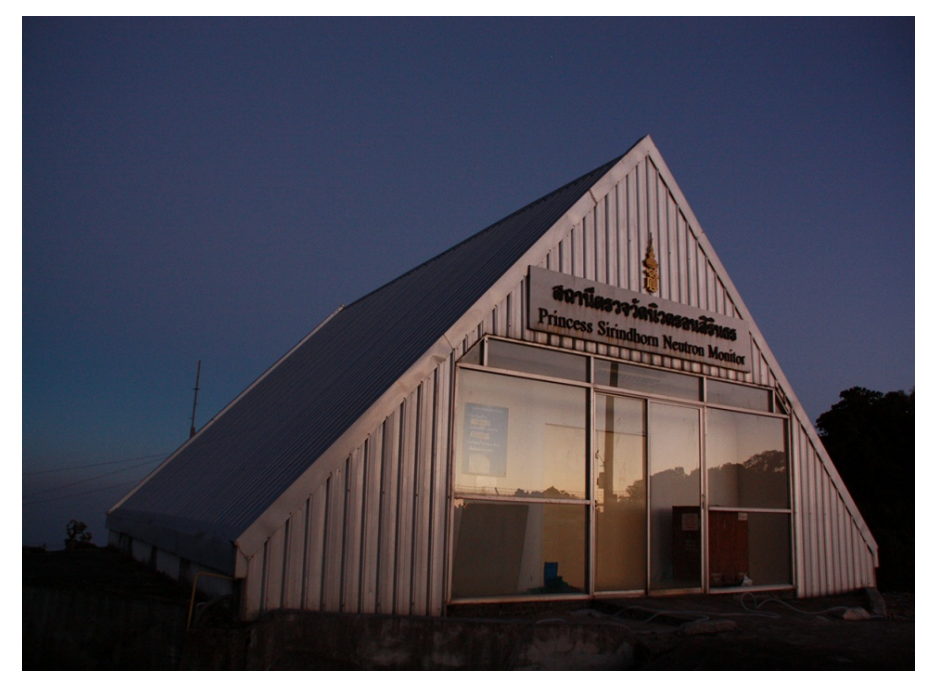

Figure 1: The Princess Sirindhorn Neutron Monitor building at the Doi Inthanon mountain summit in Chiang Mai province, Thailand.

\section{Methodology}

In order to simulate secondary cosmic rays has model atmospheric structure by the FLUKA program. A software package to simulate particle transport and interaction is based on the Monte Carlo method.[1] This project assumed that the air mass could be neglected above $51 \mathrm{~km}$ of altitude, with their accounts for only $0.04 \%$ of the atmosphere's mass. However, at high altitudes extend more than $51 \mathrm{~km}$; we can ignore the curvature of the Earth because this is less than 1\% of Earth's radius $(6378 \mathrm{~km})$. The initial model is represented in Figure 2. An atmosphere is divided into 100 layers within a rectangular box and is seated $20 \mathrm{~km}$ of horizontal length for each side. Moreover, 
we also included a chemical composition of air in the initial model (Figure 2). Using a constant molecular composition above 2000 meters with $75.512 \% \mathrm{~N}_{2}, 23.19 \% \mathrm{O}_{2}, 1.282 \% \mathrm{Ar}$, and $0.016 \%$ $\mathrm{CO}_{2}$ and included $\mathrm{H}_{2}$ in an amount that varies linearly from $0.06 \%$ by weight at sea-level to $0.01 \%$ by weight at 2000 meters.[5]

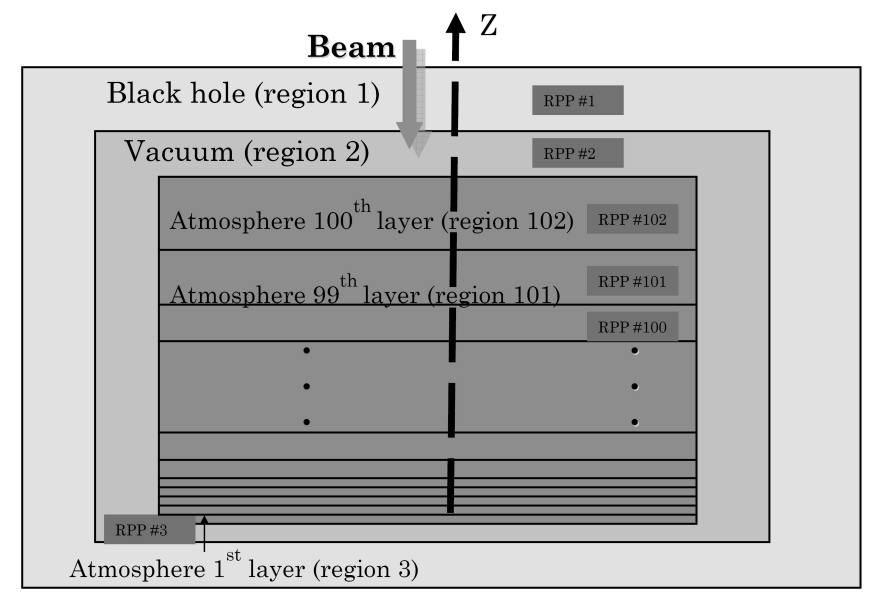

Figure 2: The atmospheric model including 100 layers of the atmosphere from sea level for the FLUKA simulation.

Noted the initial model is required sum to 100 layers because FLUKA needs the atomic composition for the simulation format by using the molecular weights for each layer. Moreover, we created the ground region at 2565 meters of altitude with granite (most of the rock at Doi Inthanon Mountain) to model the particle propagation and interactions in this region. Finally, we create initial particles as a proton beam moving vertically downward from the top of the atmosphere. Their kinetic energy was set as follows: $1,10,17,20$, and $30 \mathrm{GeV}$.

\section{Results and Discussion}

Figure 3 show the first simulation results for an atmospheric cascade from 5 mono-energetic $1 \mathrm{GeV}$ primary protons at the top of the atmosphere. The PSNM station level at 2565 meters represented by the solid blue line. In this case, we are considered in standard dry air. Each difference color point represents an interaction point of a secondary cosmic ray such as electrons, positrons, gamma rays, positive and negative muons. The results represent some of the secondary particles were absorbed before approaching the PSNM station.

The next simulation considers the Atmospheric cascade in standard dry air form 5 primary protons with energy $10 \mathrm{GeV}$; we do the same way to select the five models. Only the last model has a few secondary particles, such as gamma-ray, muon, and neutron that reach the station. (Figure 4)

For higher primary incident particles of 17,20 , and $30 \mathrm{GeV}$ in the standard dry air can produce more secondary particles and reach the PSNM station. The cascade form $30 \mathrm{GeV}$ clearly represents the number of secondary neutrons and gamma rays reach the station. (Figure 5)

On the other hand, we try to simulate a more realistic simulation to take the effect of rock, mainly granite into account, because the granite rock is the Doi Inthanon mountain's primary 
geological setting.(Figure 6) The solid blue line is the granite rock. For all incident particles of 17, 20 and $30 \mathrm{GeV}$ could not penetrate the rock, but some secondary particles reflect.
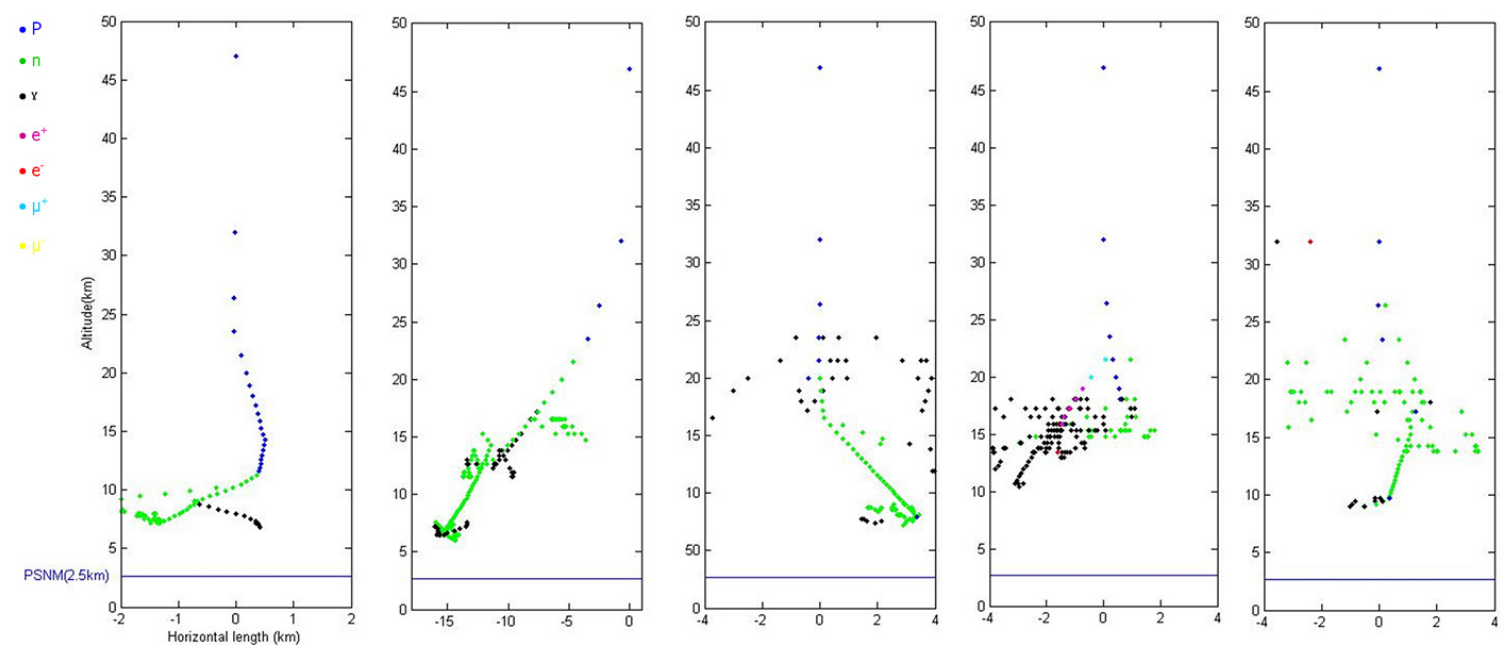

Figure 3: The atmospheric cascade form 5 primary protons which energy $1 \mathrm{GeV}$
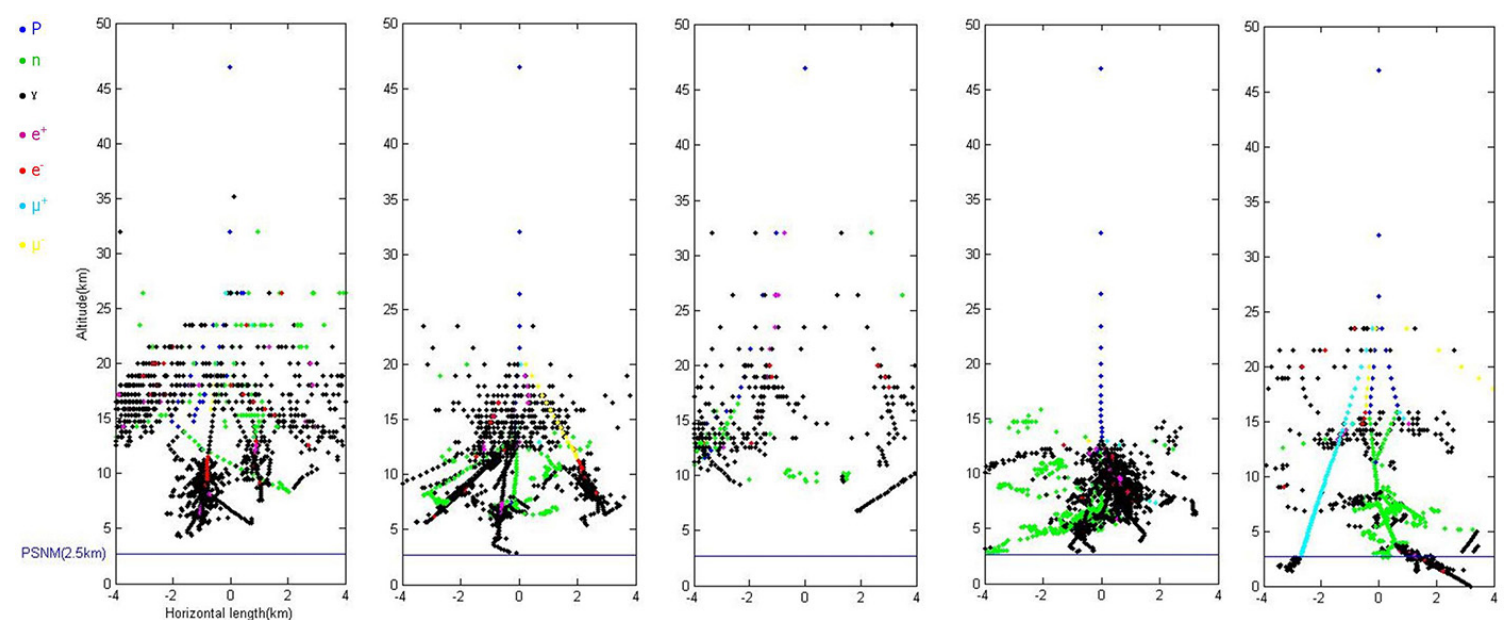

Figure 4: The atmospheric cascade form 5 primary protons which energy $10 \mathrm{GeV}$.

For discussion, we have to take action on the relationship between the neutron flux of standard dry air and the effect of granite rock on the secondary neutron. The FLUKA simulations represent both standard dry air and rock simulation with calculated only the secondary neutron flux from $17 \mathrm{GeV}$ of the primary proton at the PSNM station located on Doi Inthanon Mountain, altitude 2565 meters above sea level as shown in Figure 7. The result shows low-energy neutron flux of standard dry air (Figure 7 left) is lower than the neutron flux of rock (Figure 7 right) because some neutrons undergo spallation or reflection and lose their energy at the rock, then spraying upward to form a cloud of low-energy neutrons, which can thermalize and diffuse downward again with lower energy. The result represent consistent secondary neutron spectra at 2565 altitude for all energies considered, including a main peak at about $0.1 \mathrm{GeV}$ and a tail of low-energy neutrons about 0.001 $\mathrm{GeV}$. 

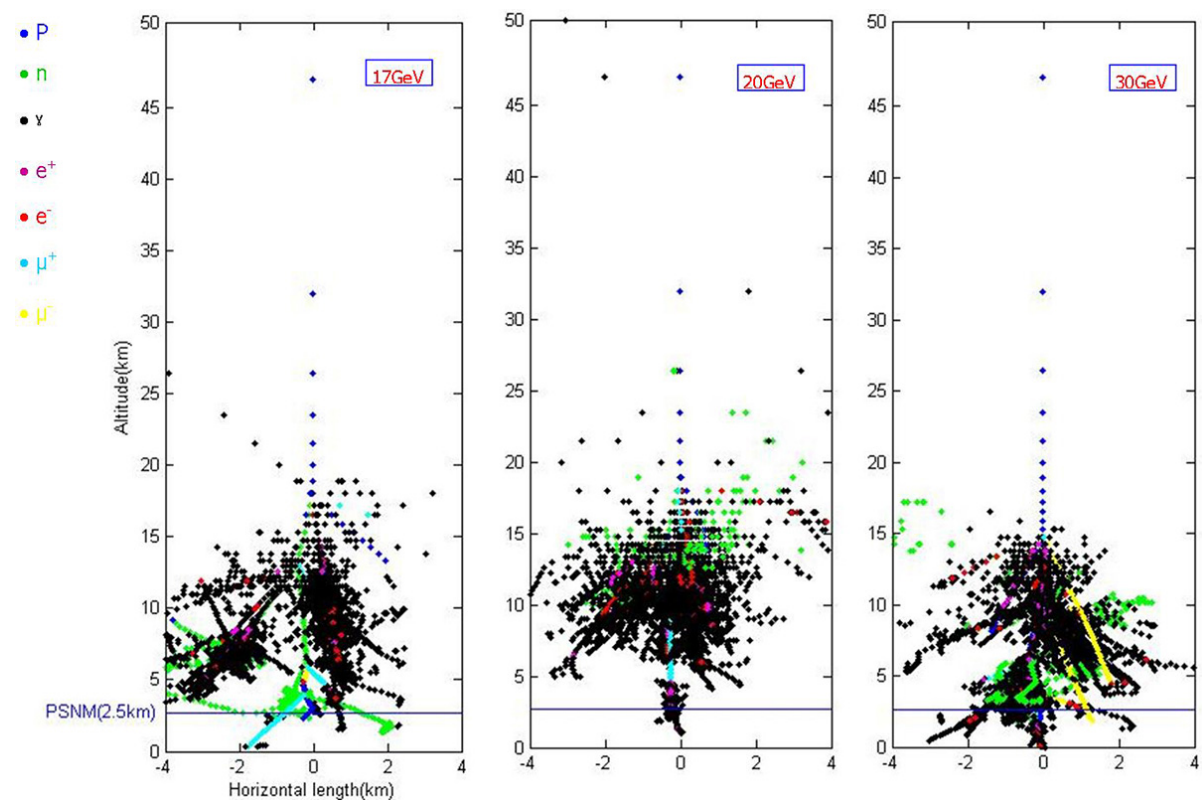

Figure 5: The cascades from 17, 20 and $30 \mathrm{GeV}$ primary protons which the impact can produce many secondary neutrons and gamma rays.
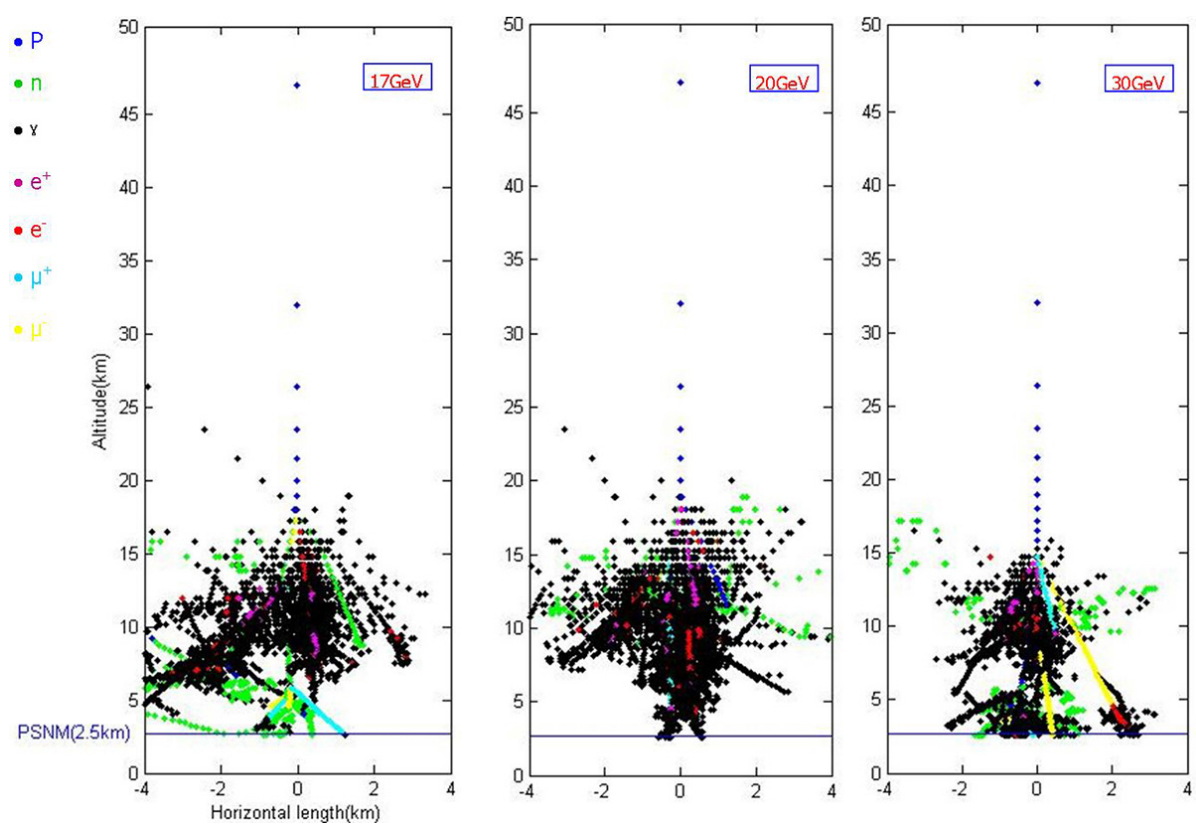

Figure 6: A more realistic simulation when we take the effect of rock (mainly granite) into account. The PSNM level are shown by a solid blue line

\section{Conclusion}

In this work, we have performed a nucleonic cascade simulation by using the FLUKA program. We developed the input files for FLUKA and simulated the effects of the secondary neutrons on air molecules by taking the effects of rock into account. The results show the expected neutron energy 
spectrum at Doi Inthanon mountain, the neutron produced by atmospheric cascade and could be detected by PSNM. This work will give us data for further analysis and a better understanding of the atmospheric structure's effects on neutron monitor counts. We will also obtain information that can be applied to improve space physics and astrophysics knowledge.

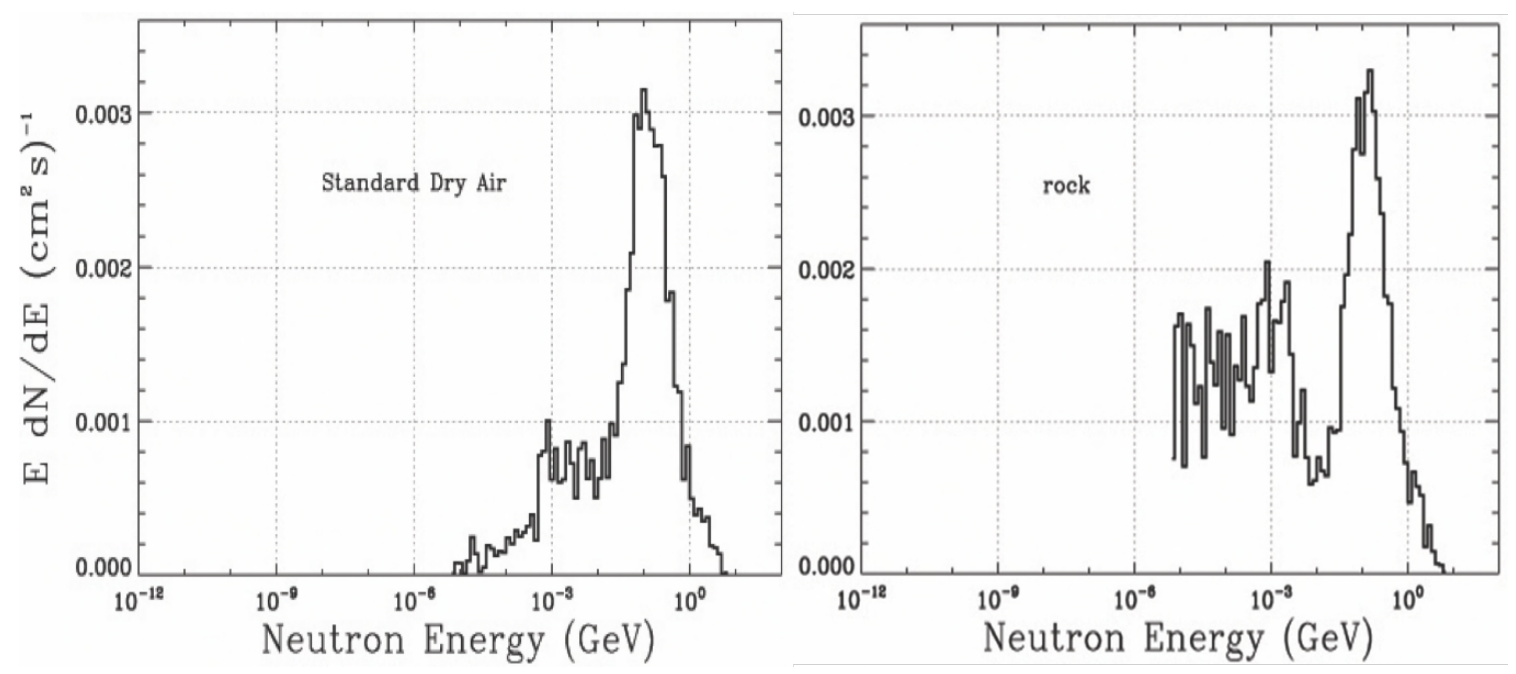

Figure 7: The secondary neutrons(left) with the standard dry air and (right) with the granite

\section{References}

[1] A. Ferrari, P. Sala, A. Fassò and J. Ranft, FLUKA: a multi-particle transport code, CERN2005-010.

[2] B. Gvozdevsky, A. Belov, R. Gushchina, E. Eroshenko, P. Kobelev, and V. Yanke,Long-Term Changes in Vertical GeomagneticCutoff Rigidities of Cosmic Rays, Physics of Atomic Nuclei 81 (2018).

[3] J. Clem, Preliminary validation of computational procedures for a new atmospheric ionizing radiation (air) model, Advances in Space Research 32 (2003).

[4] J. Tibor, Cosmogenic Nuclides: Principles, Concepts and Applications in the Earth Surface Sciences, Cambridge University Press, 2010.

[5] NOAA, NASA and USAF, U.S. Standard Atmosphere, U.S. Government Printing Office, 1976. 